\title{
Influência do Sevoflurano e do Isoflurano na Recuperação do Bloqueio Neuromuscular Produzido pelo Cisatracúrio *
}

\section{The Influence of Sevoflurane and Isoflurane on the Recovery from Cisatracurium-Induced Neuromuscular Block} Angélica de Fátima de Assunção Braga, TSA ${ }^{1}$, Franklin Sarmento da Silva Braga ${ }^{1}$,
Glória Maria Braga Potério, TSA ${ }^{1}$, Eugesse Cremonesi, TSA $^{2}$, Gislaine Mauro ${ }^{3}$

\begin{abstract}
RESUMO
Braga AFA, Braga FSS, Potério GMB, Cremenosi E, Mauro G Influência do Sevoflurano e do Isoflurano na Recuperação do Bloqueio Neuromuscular Produzido pelo Cisatracúrio
\end{abstract}

Justificativa e Objetivos - Os efeitos dos agentes bloqueadores neuromusculares sobre a junção neuromuscular são aumentados pelos anestésicos voláteis. O objetivo deste estudo foi avaliar a influência do sevoflurano e do isoflurano na recuperação do bloqueio neuromuscular produzido pelo cisatracúrio.

Método - Foram estudados 90 pacientes, estado físico ASA I e II, submetidos à cirurgias eletivas sob anestesia geral, distribuídos em três grupos: Grupo I (sevoflurano), Grupo II (isoflurano) e Grupo III (propofol). Todos os pacientes receberam como medicação pré-anestésica, midazolam $(0,1$ mg. $\mathrm{kg}^{-1}$ ) por via muscular, 30 minutos antes da cirurgia. $A$ indução anestésica foi obtida com alfentanil $\left(50 \mu \mathrm{g}_{ } \mathrm{kg}^{-1}\right)$, propofol (2,5 mg. $\left.\mathrm{kg}^{-1}\right)$ e cisatracúrio $\left(0,15 \mathrm{mg} \cdot \mathrm{kg}^{-1}\right)$. Os pacientes foram ventilados com oxigênio a 100\% sob máscara até o desaparecimento das quatro respostas a SQE, quando foram realizadas as manobras de laringoscopia e intubação traqueal. Os agente voláteis para a manutenção da anestesia foram introduzidos logo após a intubação traqueal e empregados nas concentrações de $2 \%$ e $1 \%$, respectivamente para o sevoflurano e isoflurano, e o propofol em infusão contínua ( 7 a $\left.10 \mathrm{mg} \cdot \mathrm{kg}^{-1} \cdot \mathrm{h}^{-1}\right)$. Em todos os pacientes empregou-se a mistura de oxigênio e óxido nitroso a $50 \%$. A função neuromuscular foi monitorizada por aceleromiografia do músculo adutor do polegar, empregando-se a SQE a cada 15 segundos. Foram avaliados: a duração clínica do bloqueio neuromuscular $\left(T 1_{25 \%}\right)$ e o índice de recuperação (IR= $T 1_{25-75 \%) \text {. }}$

Resultados - Os tempos médios e desvios padrão para a duração clínica $\left(T 1_{25 \%}\right)$ e índice de recuperação $\left(I R=T 1_{25-75 \%}\right)$ foram respectivamente: Grupo I (66,2 $\pm 13,42$ min e 23,6 \pm 5, 02 min), Grupo II (54,4 $\pm 6,58 \mathrm{~min}$ e 14,9 \pm 3,82 min) e Grupo III $(47,2 \pm 7,43 \mathrm{~min}$ e $16,2 \pm 2,93 \mathrm{~min})$. Em relação à duração

* Recebido do (Received from) Departamento de Anestesiologia da Faculdade de Ciências Médicas da UNICAMP, Campinas, SP

1. Professor (a) Doutor (a) do Departamento de Anestesiologia da Faculdade de Ciências Médicas da UNICAMP

2. Assessora Científica do Departamento de Anestesiologia da Faculdade de Ciências Médicas da UNICAMP

3. Anestesiologista do Hospital das Clínicas/UNICAMP

Apresentado (Submitted) em 03 de janeiro de 2002

Aceito (Accepted) para publicação em 11 de março de 2002

Correspondência para (Mail to):

Dra. Angélica de Fátima de Assunção Braga

Rua Luciano Venere Decourt, 245

Cidade Universitária

14084-040 Campinas, SP

(C) Sociedade Brasileira de Anestesiologia, 2002 clínica houve diferença significante entre os grupos I e II, I e III, e II e III. Para o índice de recuperação houve diferença significante entre o grupo I e os demais grupos.

Conclusões - A recuperação do bloqueio neuromuscular produzido pelo cisatracúrio foi mais lenta durante a anestesia com os agentes voláteis do que com o propofol, sendo o efeito mais pronunciado com o sevoflurano.

UNITERMOS - ANESTÉSICOS, Volátil: isoflurano, sevoflurano; BLOQUEADORES NEUROMUSCULARES, Não despolarizantes: cisatracúrio; MONITORIZAÇÃO: aceleromiografia

\section{SUMMARY}

Braga AFA, Braga FSS, Potério GMB, Cremenosi E, Mauro G The Influence of Sevoflurane and Isoflurane on the Recovery from Cisatracurium-Induced Neuromuscular Block

Background and Objectives - The effects of neuromuscular blockers on the neuromuscular junction are potentiated by volatile anesthetics. This study aimed at evaluating the influence of sevoflurane and isoflurane on the recovery of cisatracuriuminduced neuromuscular block.

Methods - Ninety ASA I and II patients undergoing elective surgeries under general anesthesia were included in this study. Patients were allocated in three groups: Group I (sevoflurane), Group II (isoflurane) and Group III (propofol). All patients were premedicated with intramuscular midazolam $\left(0.1 \mathrm{mg}^{\mathrm{kg}} \mathrm{k}^{-1}\right) 30$ min before surgery. Anesthesia was induced with alfentanil (50 $\left.\mu \mathrm{g} \cdot \mathrm{kg}^{-1}\right)$, propofol $\left(2.5 \mathrm{mg} \cdot \mathrm{kg}^{-1}\right)$ and cisatracurium $(0.15$ $\left.\mathrm{mg} \cdot \mathrm{kg}^{-1}\right)$. Patients were then ventilated under mask with $100 \%$ $\mathrm{O}_{2}$ until disappearance of all TOF responses when laryngoscopy and tracheal intubation were performed. Volatile agents for anesthetic maintenance were introduced immediately after tracheal intubation in $2 \%$ and $1 \%$ concentrations, respectively, for sevoflurane and isoflurane, as well as the propofol continuous infusion (7 to $10 \mathrm{mg} \cdot \mathrm{kg}^{-1} \cdot \mathrm{h}^{-1}$ ) for Group III. All patients received a $50 \%$ mixture of $\mathrm{O}_{2}$ and $\mathrm{N}_{2} \mathrm{O}$. Neuromuscular function was monitored by adductor pollicis muscle acceleromyography with TOF stimulation at 15-second intervals. Clinical duration of neuromuscular block $\left(T 1_{25 \%}\right)$ and recovery index $\left(R I=T 1_{25-75 \%}\right)$ were evaluated.

Results - Mean time and standard deviation for clinical duration $\left(T 1_{25 \%}\right)$ and recovery index $\left(R I=T 1_{25-75 \%}\right)$ were respectively: Group I (66.2 $\pm 13.42 \mathrm{~min}$ and $23.6 \pm 5.02 \mathrm{~min})$, Group II (54.4 \pm $6.58 \mathrm{~min}$ and $14.9 \pm 3.82 \mathrm{~min})$ and Group III $(47.2 \pm 7.43 \mathrm{~min}$ and $16.2 \pm 2.93 \mathrm{~min})$. There were significant differences in clinical duration between Groups I and II, I and III and II and III. There was a significant difference in recovery index between Group I and the other groups.

Conclusions - The recovery from cisatracurium-induced neuromuscular block was longer during anesthesia with volatile agents as compared to propofol. The most pronounced effect was observed with sevoflurane.

KEY WORDS - ANESTHETICS, Volatile: isoflurane, sevoflurane; MONITORING: acceleromyography; NEUROMUSCULAR BLOCKERS, Nondepolarizing: cisatracurium 


\section{INTRODUÇÃO}

$\mathrm{T}$ radicionalmente tem-se descrito que os agentes anestésicos inalatórios aumentam o efeito dos antigos bloqueadores neuromusculares (BNM) em graus variáveis, sendo esta interação tempo e dose-dependentes ${ }^{1-6}$. Este aspecto tem sido também comprovado para os novos BNM 7,8 . Um fator importante a ser considerado na ocorrência desta interação é o tipo de anestésico volátil e de BNM adespolarizante empregado, já que nem todos os agentes voláteis causam potencialização com a mesma intensidade e nem todos os BNM são potencializados da mesma maneira. Este fenômeno é menos evidente com o óxido nitroso mas alcança maior magnitude durante o uso concomitante do enflurano ou do isoflurano e diferentes BNM adespolarizantes, enquanto o halotano ocupa posição intermediária ${ }^{9,10}$. Algumas das características físicas do isoflurano e do sevoflurano que resultam em rápida captação e eliminação igualmente rápida fazem com que estes anestésicos, freqüentemente, sejam indicados em anestesias de curta duração, em especial quando realizadas em regime ambulatorial. Estudos clínicos demonstraram que, com o sevoflurano, a interação com os BNM adespolarizantes torna-se aparente em dez minutos, provavelmente devido ao seu menor coeficiente de partição músculo-gás, quando comparado com o isoflurano ${ }^{10-12}$. Nestas circunstâncias, subestimar ou ignorar a potencialização do bloqueio neuromuscular por estes agentes pode resultar em curarização inadvertida, principalmente quando do uso concomitante de BNM adespolarizantes de curta duração como o mivacúrio ou de duração de ação intermediária como o rocurônio e o cisatracúrio. A magnitude da interação cisatracúrio-agentes voláteis tem sido pouco investigada mas alguns autores relataram potencialização da depressão da resposta T1, desvio da curva dose-resposta para a esquerda e aumento significativo na recuperação do bloqueio neuromuscular ${ }^{7,13}$.

Este trabalho tem como objetivo avaliar comparativamente a influência do propofol, sevoflurano e isoflurano na recuperação do bloqueio neuromuscular produzido pelo cisatracúrio, empregado em dose única de $0,15 \mathrm{mg} \cdot \mathrm{kg}^{-1}\left(3 \times \mathrm{DE}_{95}\right)$.

\section{MÉTODO}

Após aprovação pelo Comitê de Ética do Hospital e consentimento livre e esclarecido dos pacientes, foram incluídos no estudo, 90 pacientes, estado físico ASA I e II, com idades entre 15 e 56 anos e peso entre 50 e 84 quilos, selecionados para cirurgias eletivas sob anestesia geral com indicação de intubação traqueal e ventilação controlada mecânica, distribuídos aleatoriamente por sorteio em três grupos de acordo com o agente empregado na manutenção da anestesia: Grupo I - sevoflurano $(n=30)$, Grupo II - isoflurano $(n=30)$ e Grupo III - propofol $(n=30)$.

Constituíram critérios de exclusão pacientes portadores de doenças neuromusculares, renais ou hepáticas, altera- ções hidroeletrolíticas e ácido-básicas, alterações de temperatura, sinais indicativos de dificuldades para a realização das manobras de laringoscopia e intubação traqueal Mallampati 3 e $4^{14}$, história de refluxo gastroesofágico e em uso de drogas que interagem com bloqueadores neuromusculares.

A medicação pré-anestésica consistiu de midazolam $(0,1$ $\mathrm{mg} \cdot \mathrm{kg}^{-1}$ ), por via muscular, 30 minutos antes da indução anestésica. Na sala cirúrgica, uma veia periférica foi canulizada para hidratação e administração de drogas. A indução foi obtida com alfentanil $\left(50 \mu \mathrm{g} . \mathrm{kg}^{-1}\right)$; seguido de propofol $\left(2,5 \mathrm{mg} \cdot \mathrm{kg}^{-1}\right)$ e cisatracúrio $\left(0,15 \mathrm{mg} \cdot \mathrm{kg}^{-1}\right)$. Os pacientes foram ventilados sob máscara com oxigênio a $100 \%$, realizando-se a laringoscopia e a intubação traqueal, quando do desaparecimento das quatro respostas à seqüência de quatro estímulos (SQE). Nos grupos I e II, o agente volátil para a manutenção da anestesia foi introduzido logo após a intubação traqueal, através de vaporizador calibrado e mantida a concentração de $2 \%$ e $1 \%$ para sevoflurano e isoflurano, respectivamente, até a recuperação de $75 \%$ da resposta de $\mathrm{T} 1$; no Grupo III foi iniciada a infusão contínua de propofol (7 a 10 $\left.\mathrm{mg} \cdot \mathrm{kg}^{-1} \cdot \mathrm{h}^{-1}\right)$. Em todos os pacientes empregou-se mistura de oxigênio e óxido nitroso a $50 \%$. Doses adicionais de fentanil $\left(2 \mu \mathrm{g} \cdot \mathrm{kg}^{-1}\right)$, foram administradas na vigência de sinais clínicos de anestesia superficial (pressão arterial e freqüência cardíaca maiores do que os valores basais). Os pacientes foram ventilados mecanicamente para manter a $\mathrm{P}_{\mathrm{ET}} \mathrm{CO}_{2}$ entre 32 e $36 \mathrm{mmHg}$.

Utilizou-se como monitorização contínua o cardioscópio na derivação $D_{\| I}$, oxímetro de pulso, capnógrafo, monitor não invasivo de pressão arterial, e aceleromiografia para avaliação do bloqueio neuromuscular. Foi medida a temperatura da pele sobre o músculo monitorizado (região tenar) que se manteve acima de $32^{\circ} \mathrm{C}$.

Antes da indução anestésica foram aplicados estímulos supramaximais $(1 \mathrm{~Hz})$, durante cinco minutos, para a estabilização da resposta neuromuscular do adutor do polegar, empregando-se eletrodos de superfície no trajeto do nervo ulnar, no punho. Um transdutor de aceleração (piezoelétrico), foi fixado na falange distal do polegar do membro monitorizado. Após a administração do opióide e do propofol e antes da administração do cisatracúrio, a estimulação do nervo ulnar foi continuada empregando-se a SQE a cada 15 segundos. Nos registros das respostas do adutor do polegar (Figura 1), observam-se: 1) momento da indução anestésica; 2 ) início da administração do agente volátil (traçado superior - sevoflurano; traçado médio - isoflurano) ou propofol (traçado inferior); 3) duração clínica do bloqueio neuromuscular $\left(\mathrm{T}_{2}{ }_{25}\right)$ - intervalo de tempo em minutos, decorrido entre a administração do cisatracúrio e a recuperação de $25 \%$ da primeira resposta à SQE; 4 ) índice de recuperação (IR= $\left.\mathrm{T} 1_{25-75 \%}\right)$ - intervalo de tempo em minutos para a recuperação de $25 \%$ até $75 \%$ da altura da primeira resposta à SQE.

Para a análise estatística dos dados demográficos e farmacodinâmicos empregaram-se ANOVAe teste $t$ de Student pareado, adotando-se nível de significância $5 \%(p<0,05)$. 


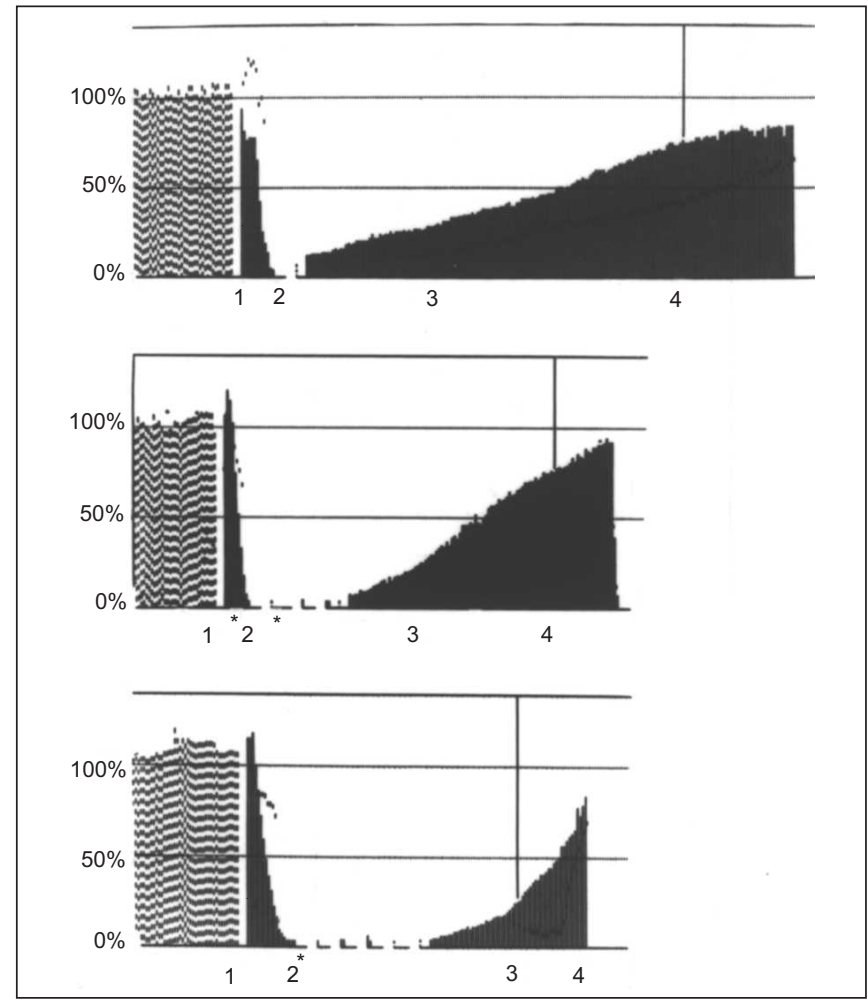

Figura 1 - Respostas do Músculo Adutor do Polegar (Aceleromiografia); traçado superior (sevoflurano - Grupo I); traçado médio (isoflurano - Grupo II); traçado inferior (propofol - Grupo III). 1 - indução anestésica; 2 - início da administração do agente volátil ou infusão contínua de propofol; 3- duração clínica $\left(\mathrm{T}_{125 \%}\right)$; 4- índice de recuperação $\left(\mathrm{IR}=\mathrm{T}_{125-75 \%}\right)$

\section{RESULTADOS}

Com relação aos dados demográficos dos pacientes, não houve diferença significativa entre os grupos, sendo os mesmos considerados uniformes; o mesmo foi observado para a temperatura da região tenar e a $\mathrm{P}_{\mathrm{ET}} \mathrm{CO}_{2}$ (Tabela I).
A duração clínica $\left(\mathrm{T}_{25 \%}\right)$ do bloqueio produzido pelo cisatracúrio foi mais prolongada nos grupos I (sevoflurano) e II (isoflurano) do que no grupo III (propofol), com diferença significante entre os grupos I e II, I I III e II e III; O índice de recuperação ( $\left.\mathrm{T} 1_{25 \%-75 \%}\right)$ foi significantemente mais longo com o sevoflurano em relação ao isoflurano e propofol (Tabela II). Em ambos os grupos, no per-operatório, houve necessidade de dose adicional de fentanil que variou de 100 a $500 \mu \mathrm{g}$, empregada em 12, 11 e 25 pacientes nos grupos I, II e III, respectivamente.

\section{DISCUSSÃo}

Estudos dose-resposta têm sido freqüentemente realizados para estimar e comparar a potência dos BNM adespolarizantes durante anestesia. Trabalhos anteriores demonstraram potencialização dos efeitos destas drogas pelos agentes voláteis, mesmo quando são empregadas pequenas concentrações, resultando em aumento na recuperação do bloqueio neuromuscular, com conseqüente redução do consumo de BNM adespolarizante ${ }^{1-5,8,10,11,15,16}$.

São poucos os estudos da magnitude deste efeito na curva dose-resposta do cisatracúrio, isômero do atracúrio com menos efeitos colaterais em relação ao seu antecessor ${ }^{15}$. Um estudo mostrou que as $D_{50}$ e $D E_{95}$ do cisatracúrio são significativamente menores com os agentes voláteis do que com o propofol ${ }^{13}$, sugerindo que o bloqueio neuromuscular produzido pelo cisatracúrio é influenciado pelos agentes voláteis. Anteriormente, outros autores observaram redução na dose de cisatracúrio em cerca de $42 \%, 41 \%$ e $60 \%$ quando do emprego de isoflurano, sevoflurano e desflurano, respectivamente, em comparação ao propofol em infusão contínua na dose de 6 a $8 \mathrm{mg} \cdot \mathrm{kg}^{-1} \cdot \mathrm{h}^{-1}$, sendo esta redução significativamente maior com o desflurano ${ }^{17}$.

O mecanismo de ação desta interação ainda não está totalmente esclarecido. Alguns efeitos que podem favorecer a potencialização já foram bem estabelecidos, e incluem a ação dos agentes voláteis nos canais dos receptores para

Tabela I - Características Demográficas dos Pacientes, Valores de Temperatura da Região Tenar e Pressão de $\mathrm{CO}_{2}$ Expirado

\begin{tabular}{|c|c|c|c|}
\hline & Grupo I & Grupo II & Grupo III \\
\hline Idade (anos) * & $35 \pm 6,72$ & $34,7 \pm 8,73$ & $38,4 \pm 11,33$ \\
\hline Peso $(\mathrm{kg})$ * & $60,1 \pm 4,25$ & $61,5 \pm 4,69$ & $62,2 \pm 7,08$ \\
\hline Estado físico (ASA I:II) & 28:02 & 29:01 & $28: 02$ \\
\hline Temperatura região tenar $\left({ }^{\circ} \mathrm{C}\right)$ * & $33,4 \pm 0,59$ & $33,5 \pm 0,57$ & $33,3 \pm 0,57$ \\
\hline $\mathrm{P}_{\mathrm{ET}} \mathrm{CO}_{2}(\mathrm{mmHg})$ * & $34,7 \pm 0,3$ & $34,5 \pm 0,5$ & $34,3 \pm 0,3$ \\
\hline
\end{tabular}

* Dados expressos pela Média \pm DP

Tabela II - Parâmetros Neuromusculares (Média \pm DP)

\begin{tabular}{|c|c|c|c|}
\hline & $\begin{array}{c}\text { Grupo I } \\
\text { (sevoflurano) }\end{array}$ & $\begin{array}{c}\text { Grupo II } \\
\text { (isoflurano) }\end{array}$ & $\begin{array}{c}\text { Grupo III } \\
\text { (propofol) }\end{array}$ \\
\hline Duração clínica $\left(T 1_{25 \%}\right)$ - min * & $66,2 \pm 13,42$ & $54,4 \pm 6,58$ & $47,2 \pm 7,43$ \\
\hline Índice de recuperação ** $\left(T 1_{25-75 \%}\right)-$ min & $23,6 \pm 5,02$ & $14,9 \pm 3,82$ & $16,2 \pm 2,93$ \\
\hline
\end{tabular}

* GI \# GII e GIII; GII \# GIII (p<0,05); ** GI \# GII e GIII $(p<0,05)$ 
acetilcolina com conseqüente alteração conformacional destes canais, ação no sistema nervoso central, causando depressão reflexa medular e contribuindo para a redução do tônus com relaxamento muscular esquelético, diminuição da sensibilidade da membrana pós-juncional à despolarização causada pela acetilcolina e aumento do fluxo sangüíneo muscular, possibilitando que maior quantidade de bloqueador neuromuscular chegue ao local de ação ${ }^{18-22}$.

Estudos da interação entre os BNM e os anestésicos voláteis levam em consideração um período de 30 a 45 minutos necessário para estes agentes difundirem-se para o compartimento muscular e, assim, atingir o equilíbrio entre as concentrações inaladas/alveolares/ plasmáticas/ musculares, antes da administração dos BNM adespolarizante ${ }^{2,4,5,10,23}$. Os resultados assim obtidos são de relevância discutível quando transpostos para a clínica, pois distanciam-se dos modelos clínicos nos quais a dose curarizante é, quase sempre, aplicada antes da intubação traqueal, ou seja, na ausência dos halogenados ${ }^{24,25}$. Assim, neste estudo, buscou-se seguir o modelo de uma anestesia de rotina e seguiu-se a seqüência habitual de emprego das drogas, optando-se pela dose de cisatracúrio de $0,15 \mathrm{mg} \cdot \mathrm{kg}^{-1}$.

A análise de T1 possibilita avaliar a regressão do bloqueio neuromuscular pelo índice de recuperação ( $\mathrm{T} 1_{25-75 \%}$ ), que representa um padrão de comparação de acordo com a dose total de BNM empregada e correlaciona-se de maneira inversa com a eliminação da droga ${ }^{26}$.

Os $I_{25-75}$, de diferentes BNM adespolarizantes são: 6 a 8 minutos para o mivacúrio; 10 a 15 minutos para o atracúrio, o vecurônio, rocurônio e para o cisatracúrio; 35 minutos para o pipecurônio e 40 minutos para o doxacúrio ${ }^{27}$. Estes índices podem ser afetados pelo uso contínuo, doses repetidas ou altas doses de BNM adespolarizantes e por outras drogas cuja redistribuição interfira com a regressão do bloqueio neuromuscular ${ }^{28,29}$.

O valor médio do $\mathrm{IR}_{25-75}$ após simples dose do cisatracúrio foi significativamente prolongado durante anestesia com sevoflurano (23,6 $\pm 5,02$ minutos) quando comparado ao isoflurano $(14,9 \pm 3,82$ minutos) ou propofol (16,2 $\pm 2,93$ minutos). Isto pode ser devido em parte à variabilidade interindividual ter sido maior durante a anestesia com sevoflurano. Também pode ser inversamente relacionado à potência analgésica e hipnótica ou à solubilidade sangüínea dos agentes inalatórios, no entanto estas hipóteses necessitam ser melhor estudadas ${ }^{30}$. Estes resultados são semelhantes aos descritos anteriormente para o rocurônio ${ }^{8,30}$, mas contrários para o pancurônio, vecurônio e atracúrio, nos quais os $I R_{25-75}$ foram igualmente prolongados pelo sevoflurano e isoflurano ${ }^{2}$.
Embora os valores de índice de recuperação e duração clínica por nós obtidos, nos três grupos, tenham sido maiores do que os relatados por Wulf e col. ${ }^{13}$, estes também encontraram IR $\mathrm{R}_{25-75}$ significativamente mais longo com o sevoflurano do que com o isoflurano e propofol. A duração clínica e o índice de recuperação dependem da dose empregada; portanto, uma provável razão para a maior duração pode ter sido a dose maior de cisatracúrio empregada neste estudo. Outros autores, mesmo empregando doses semelhantes de cisatracúrio, obtiveram tempos menos prolongados $(46,6 \pm 8$ $\min )^{31}$ ou mesmo semelhantes $(68,3 \pm 2,4 \mathrm{~min})^{32}$ quando a dose foi aumentada para $4 \times \mathrm{DE}_{95}$. Outro fator que pode ter contribuído para a maior duração do bloqueio é a manutenção do anestésico volátil, nas concentrações padronizadas, durante a regressão, até a recuperação de $75 \%$ da altura da primeira resposta à seqüência de quatro estímulos. Ao final das anestesias, o anestésico inalatório é interrompido alguns minutos antes da descurarização. Como o coeficiente de partição sangue-gás e músculo-gás do sevoflurano é menor do que o do isoflurano, é possível que o equilíbrio entre a concentração inspirada e na junção neuromuscular seja alcançada mais rapidamente com este agente do que com outros, exceto o desflurano, o qualé menos solúvel no sangue e nos tecidos do que o sevoflurano ${ }^{10,11,33,34}$. Gill e col. ${ }^{35}$ avaliaram a regressão do bloqueio produzido pelo atracúrio em pacientes que estavam inalando concentrações inspiradas de enflurano de $1 \%$ e $2 \%$ e constataram tempos de recuperação maiores do que quando o enflurano foi suspenso no momento da reversão. Os efeitos de concentrações residuais de isoflurano sobre a regressão do bloqueio produzido pelo vecurônio foram estudados, comparativamente, em pacientes que continuaram inalando isoflurano a 1,5\% durante a reversão do bloqueio e em outros nos quais houve interrupção do halogenado 15 minutos antes da neostigmina. Estes resultados indicaram que a recuperação do bloqueio residual pode ser prolongada pelos anestésicos halogenados ${ }^{36}$.

Considerando que os halogenados prolongam a duração do bloqueio produzido pelo cisatracúrio, alguns aspectos tornam-se relevantes durante as anestesias de curta duração. Não há necessidade de diminuir a dose inicial do BNM, no entanto para orientação quanto à injeção de doses adicionais e para evitar o risco de curarização residual, recomenda-se a monitorização com estimuladores de nervo periférico.

O isoflurano e o sevoflurano prolongam a duração do bloqueio neuromuscular produzido pelo cisatracúrio, quando comparado com o propofol. Este efeito é mais pronunciado com o sevoflurano e evidenciado pela maior duração clínica e maior índice de recuperação do bloqueio com este agente. 


\section{The Influence of Sevoflurane and Isoflurane on the Recovery from Cisatracurium- Induced Neuromuscular Block}

Angélica de Fátima de Assunção Braga, TSA, M.D., Franklin Sarmento da Silva Braga, M.D., Glória Maria Braga Potério, TSA, M.D., Eugesse Cremonesi, TSA, M.D., Gislaine Mauro, M.D.,

\section{INTRODUCTION}

It has been traditionally described that inhalational anesthetics potentiate the effects of classic neuromuscular blockers (NMB) in different degrees and in a time and dose-dependent manner ${ }^{1-6}$. This has also been shown for new NMBs ${ }^{7-8}$. An important factor for such interaction is the type of volatile anesthetics and nondepolarizing NMB, since not all volatile agents have the same potentiation intensity and not all NMBs are equally potentiated. This phenomenon is less evident with nitrous oxide but is more intense with the simultaneous use of enflurane or isoflurane and different nondepolarizing NMBs, while halothane exhibits an intermediate effect ${ }^{9,10}$. Isoflurane and sevoflurane physical properties which result in fast uptake and excretion have led to their frequent indication for short anesthesias, especially for outpatient procedures. Clinical studies have shown that, with sevoflurane, the interaction with nondepolarizing NMBs is apparent in 10 minutes, probably due to its low muscle-gas partition coefficient, as compared to isoflurane ${ }^{10-12}$. In those circumstances, underestimate or ignore neuromuscular blocker potentiation induced by such agents may result in inadvertent curarization, especially when short duration nondepolarizing NMBs, such as mivacurium, or intermediate duration NMBs, such as rocuronium and cisatracurium, are used. The magnitude of cisatracurium/volatile agents interaction has been poorly investigated, but some authors have reported potentiation of T1 response depression, dose-response curve shift to the left and significant increase in neuromuscular block recovery time ${ }^{7,13}$

The aim of this study was to comparatively evaluate the influence of propofol, sevoflurane and isoflurane in cisatracuriuminduced neuromuscular block in a single dose of $0.15 \mathrm{mg} . \mathrm{kg}^{-1}$ $\left(3 \times E D_{95}\right)$.

\section{METHODS}

After the Hospital's Ethical Committee approval and with informed consents, 90 patients physical status ASA I and II, aged 15 to 56 years, weighing 50 to $84 \mathrm{~kg}$, scheduled for elective surgeries under general anesthesia with tracheal intubation and controlled mechanical ventilation, were randomly distributed in three groups according to the agent used for anesthetic maintenance: Group I - sevoflurane $(n=30)$, Group II - isoflurane $(n=30)$ and Group III - propofol $(n=30)$.
Exclusion criteria were neuromuscular, renal or liver diseases, hydroelectrolytic and acid-base disturbances, changes in temperature, Mallampati rates 3 and $4{ }^{14}$, history of esophageal reflux and drugs interacting with neuromuscular blockers.

Patients were premedicated with intramuscular midazolam $\left(0.1 \mathrm{mg}^{\mathrm{kg}}{ }^{-1}\right) 30$ minutes before anesthesia. A peripheral vein was catheterized in the operating room for hydration and drug administration. Anesthesia was induced with alfentanil $\left(50 \mu \mathrm{g} \cdot \mathrm{kg}^{-1}\right)$, followed by propofol $\left(2.5 \mathrm{mg} \cdot \mathrm{kg}^{-1}\right)$ and cisatracurium $\left(0.15 \mathrm{mg} \cdot \mathrm{kg}^{-1}\right)$. Patients were ventilated with $100 \%$ oxygen under mask until laryngoscopy and tracheal intubation maneuvers, performed after the disappearance of all TOF stimulation responses. In Groups I and II, volatile agents for anesthetic maintenance were introduced immediately after tracheal intubation through a gaged vaporizer, maintaining concentrations of $2 \%$ and $1 \%$ for sevoflurane and isoflurane, respectively, until $75 \%$ recovery of $\mathrm{T} 1$ response; Group III received propofol continuous infusion (7 to $\left.10 \mathrm{mg} \cdot \mathrm{kg}^{-1} \cdot \mathrm{h}^{-1}\right)$. All patients received a $50 \%$ mixture of oxygen and nitrous oxide. Additional fentanyl doses $\left(2 \mu \mathrm{g} . \mathrm{kg}^{-1}\right)$ were administered in the presence of clinical signs of superficial anesthesia (blood pressure and heart rate increases above baseline values). Patients were mechanically ventilated to maintain $\mathrm{P}_{\mathrm{ET}} \mathrm{CO}_{2}$ between 32 and $36 \mathrm{mmHg}$.

Continuous monitoring consisted of cardioscope in $D_{\text {II }}$ lead, pulse oximetry, capnography, non-invasive blood pressure and acceleromyography to evaluate neuromuscular block. Skin temperature was measured over the monitored muscle (thenal region) and was maintained above $32^{\circ} \mathrm{C}$.

Supramaximal stimulations $(1 \mathrm{~Hz})$ were applied for 5 minutes before anesthetic induction to stabilize adductor pollicis neuromuscular response, using surface electrodes on the ulnar nerve pathway on the wrist. An acceleration transducer (piezoelectric) was fixed on the thumb's distal phalange of the monitored limb. After opioid and propofol but before cisatracurium administration, ulnar nerve was continuously stimulated with TOF at 15 -second intervals. Adductor pollicis responses (Figure 1) recorded: 1) anesthetic induction time; 2) beginning of volatile agent administration (upper tracing sevoflurane; medium - tracing - isoflurane) or propofol (lower tracing); 3) neuromuscular blocker clinical duration ( $\left.\mathrm{T} 1_{25 \%}\right)$ time in minutes before cisatracurium administration and $25 \%$ recovery of first TOF response; 4 ) recovery index (RI = $\mathrm{T} 1_{25-75 \%}$ ) - time in minutes for $25 \%$ to $75 \%$ recovery of first TOF response.

ANOVA and Student's $t$ paired tests were used for statistical analysis of demographics and pharmacodynamic data, considering significant $p<0.05(5 \%)$.

\section{RESULTS}

There were no significant differences between groups in demographics, thenal region temperature and $\mathrm{P}_{\mathrm{ET}} \mathrm{CO}_{2}$ ( Table I). 


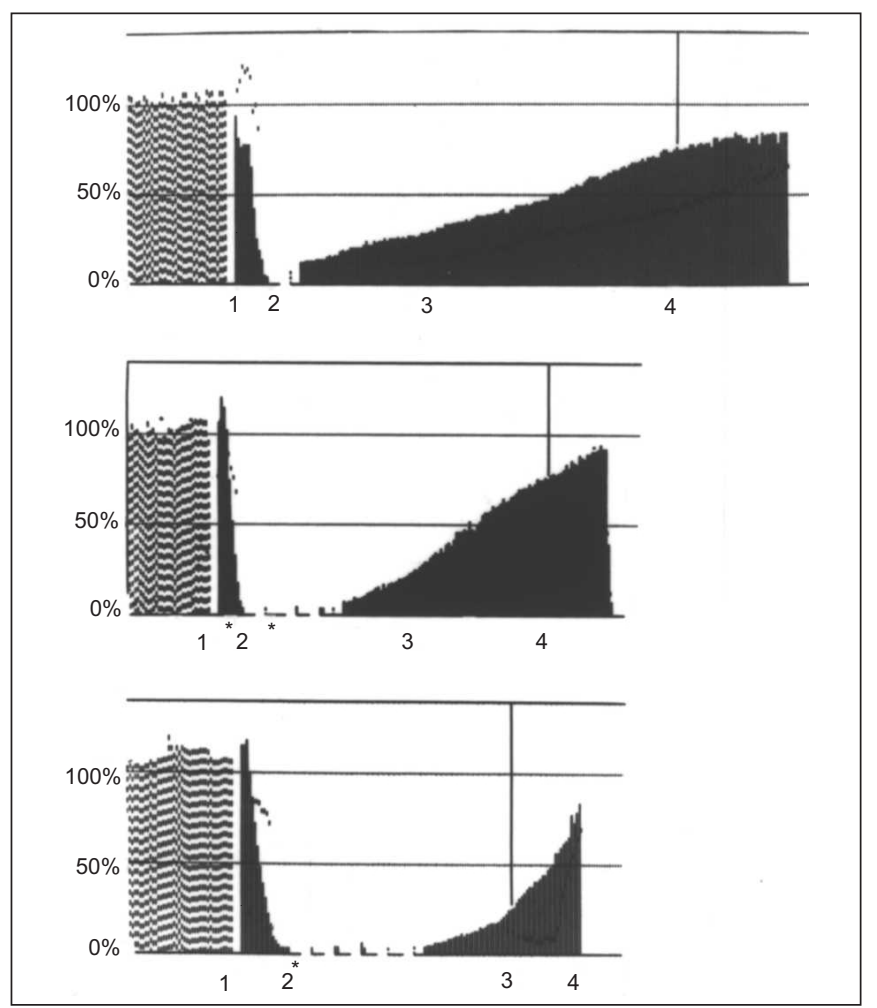

Figure 1 - Adductor pollicis muscle responses (Acceleromyography); upper tracing (sevoflurane - Group I); medium tracing (isoflurane - Group II); lower tracing (propofol - Group III). 1anesthetic induction; 2- beginning of volatile agent or propofol continuous infusion; 3 - clinical duration $\left(\mathrm{T}_{2} 2 \%\right.$ ); 4 - recovery in$\operatorname{dex}\left(\mathrm{RI}=\mathrm{T} 1_{25-75 \%}\right)$

Cisatracurium-induced clinical blockade duration $\left(\mathrm{T} 1_{25 \%}\right)$ was longer in groups I (sevoflurane) and II (isoflurane) as compared to group III (propofol), with significant differences between groups I and II, I and III, and II and III. Recovery in$\operatorname{dex}\left(\mathrm{T}_{25-75 \%}\right)$ was significantly longer with sevoflurane as compared to isoflurane and propofol (Table II).

All groups needed additional intraoperative fentanyl doses, which varied from 100 to $500 \mu \mathrm{g}$, and were administered to 12,11 and 25 patients of groups I, II and III, respectively.

\section{DISCUSSION}

Dose-response studies have been often carried out to estimate and compare nondepolarizing NMBs potency during anesthesia. Previous studies have shown potentiation of such drugs' effects by volatile agents, even when used in low concentrations, resulting in longer neuromuscular block recovery with consequent decrease in nondepolarizing NMBs consumption 1-5,8,10-11,15-16.

There are few studies on the magnitude of such effect in the dose-response curve of cisatracurium which is an atracurium isomer with less side-effects ${ }^{15}$. A study has shown that cisatracurium $E D_{50}$ and $E D_{95}$ are significantly lower with volatile agents as compared to propofol ${ }^{13}$, suggesting that cisatracurium-induced neuromuscular block is influenced by volatile agents. Other authors had already observed cisatracurium dose decrease in approximately $42 \%, 41 \%$ and $60 \%$ with isoflurane, sevoflurane and desflurane, respectively, as compared to propofol continuous infusion (6 to $\left.8 \mathrm{mg} \cdot \mathrm{kg}^{-1} \cdot \mathrm{h}^{-1}\right)$, being this decrease significantly higher with desflurane ${ }^{17}$.

The mechanism of such interaction is still not totally known. Some effects which may favor potentiation have already been established and include the action of volatile agents producing conformational changes in acetylcholine receptor channels in the central nervous system, causing reflex medullary depression and contributing for decreased tonus and skeletal muscle relaxation. There are also a decreased post-junctional membrane sensitivity to the depolarization caused by acetylcholine and increased muscle blood flow, allowing a larger amount of neuromuscular blocker to reach the action site ${ }^{18-22}$.

Studies on the interaction of NMBs and volatile agents take into consideration a period of 35 to 45 minutes, necessary for these agents to spread to the muscle compartment and reach a balance between inhaled/alveolar/plasma/muscle concentrations before nondepolarizing NMB administration $^{2,4,5,10,23}$. Results obtained are questionable when transported to clinical settings, because they are distant from clinical models were the curarizing dose is almost always administered before tracheal intubation, that is, in the ab-

Table I - Demographics Data, Thenal Region Temperature and Expired $\mathrm{CO}_{2}$ Pressure

\begin{tabular}{|c|c|c|c|}
\hline & Group I & Group II & Group III \\
\hline Age (years) * & $35 \pm 6.72$ & $34.7 \pm 8.73$ & $38.4 \pm 11.33$ \\
\hline Weight $(\mathrm{kg})$ * & $60.1 \pm 4.25$ & $61.5 \pm 4.69$ & $62.2 \pm 7.08$ \\
\hline Physical status (ASA I:II) & 28:02 & 29:01 & 28:02 \\
\hline Thenal region temperature $\left({ }^{\circ} \mathrm{C}\right)$ * & $33.4 \pm 0.59$ & $33.5 \pm 0.57$ & $33.3 \pm 0.57$ \\
\hline $\mathrm{P}_{\mathrm{ET}} \mathrm{CO}_{2}(\mathrm{mmHg})$ * & $34.7 \pm 0.3$ & $34.5 \pm 0.5$ & $34.3 \pm 0.3$ \\
\hline
\end{tabular}

Table II - Neuromuscular Parameters (Mean \pm SD)

\begin{tabular}{lccc}
\hline & $\begin{array}{c}\text { Group I } \\
\text { (sevoflurane) }\end{array}$ & $\begin{array}{c}\text { Group II } \\
\text { (isoflurane) }\end{array}$ & $\begin{array}{c}\text { Group III } \\
\text { (propofol) }\end{array}$ \\
\hline Clinical duration $\left(T 1_{25 \%}\right)-$ min* $^{*}$ & $66.2 \pm 13.42$ & $54.4 \pm 6.58$ & $47.2 \pm 7.43$ \\
Recovery index ${ }^{* *}\left(T 1_{25-75 \%}\right)-\min$ & $23.6 \pm 5.02$ & $14.9 \pm 3.82$ & $16.2 \pm 2.93$ \\
\hline
\end{tabular}

${ }^{*} \mathrm{GI}$ \# GII and GIII; GII \# GIII (p<0.05); ** GI \# GII and GIII ( $\left.<<0.05\right)$ 
sence of halogenate agents ${ }^{24,25}$. So, in our study, we tried to follow the model of a routine anesthesia, with the normal sequence of drugs administration and a $0.15 \mathrm{mg} \cdot \mathrm{kg}^{-1}$ cisatracurium dose.

The analysis of T1 provides an evaluation of neuromuscular block regression by the recovery index $\left(T 1_{25-75}\right)$, which is a comparison standard according to the total NMB dose and is inversely correlated to drug excretion ${ }^{26}$.

$\mathrm{RI}_{25-75}$ of different NMB nondepolarizing agents are: 6 to 8 minutes for mivacurium; 10 to 15 minutes for atracurium, vecuronium, rocuronium and cisatracurium; 35 minutes for pipecuronium and 40 minutes for doxacurium ${ }^{27}$. These index may be affected by continuous use, repeated doses or high nondepolarizing NMB doses, and by other drugs interfering with neuromuscular block recovery in their redistribution 28,29

Mean $\mathrm{RI}_{25-75}$ value after a single cisatracurium dose was significantly longer during sevoflurane anesthesia (23.6 \pm 5.02 minutes) compared to isoflurane (14.9 \pm 3.82 minutes) or propofol (16.2 \pm 2.93 minutes). This may be partly due to a higher individual variation during anesthesia with sevoflurane. It may also be inversely related to analgesic and hypnotic potency or to blood solubility of inhalational agents, but such hypotheses need further studies ${ }^{30}$. These results are similar to those previously reported for rocuronium ${ }^{8,30}$, different from those reported for pancuronium, vecuronium and atracurium, where $\mathrm{RI}_{25-75}$ were equally prolonged by sevoflurane and isoflurane ${ }^{2}$. Although recovery index and clinical duration values found in our study for the three groups were longer than those reported by Wulf et al. ${ }^{13}$, they have also found $\mathrm{RI}_{25-75}$ significantly longer with sevoflurane as compared to isoflurane and propofol. Clinical duration and recovery index are dose-dependent. Therefore, a likely reason for the longer duration could be the higher cisatracurium dose used in our study. Other authors, even using similar cisatracurium doses, have found shorter $(46.6 \pm 8 \text { minutes })^{31}$ or even similar times $(68.3 \pm 2.4 \text { minutes })^{32}$ when the dose was increased to $4 \times \mathrm{DE}_{95}$. Another factor which might have contributed for the longer blockade duration is the maintenance of volatile anesthetics in standardized concentrations during regression, until $75 \%$ recovery of the first TOF response. At anesthesia completion, the inhalational agent is withdrawn some minutes before decurarization. Since the blood-gas and muscle-gas partition coefficients of sevoflurane are lower then those of isoflurane, it is possible that the balance between inspired and neuromuscular junction concentrations is more rapidly reached with such agent than with others, except for desflurane, which is less soluble in blood and tissues than sevoflurane ${ }^{10,11,33,34}$. Gill et al. ${ }^{35}$ have evaluated atracurium-induced blockade regression in patients inhaling $1 \%$ and $2 \%$ enflurane concentrations and have observed longer recovery times than when enflurane was withdrawn at the moment of reversion. Residual isoflurane concentration effects on vecuronium-induced blockade regression were comparatively studied in patients who continued inhaling $1.5 \%$ isoflurane during blockade reversion and those in whom the agent was withdrawn 15 minutes before neostigmine. These studies have indicated that residual block recovery may be prolonged by halogenate agents ${ }^{36}$.

Considering that halogenate agents prolong cisatracuriuminduced blockade, some aspects are relevant during short-duration anesthesias. There is no need to decrease initial NMB dose. However, peripheral nerve stimulator monitoring is recommended for the injection of additional doses and to prevent residual curarization risks.

Isoflurane and sevoflurane prolong cisatracurium-induced neuromuscular block duration as compared to propofol. This effect is even more pronounced with sevoflurane and confirmed by longer clinical duration and higher recovery index.

\section{REFERÊNCIAS - REFERENCES}

01. Rupp SM, Miller RD, Gencarelli PJ - Vecuronium - induced neuromuscular blockade during enflurane, isoflurane, and halothane anesthesia in humans. Anesthesiology, 1984;60: 102-105.

02. Vanlinthout LEH, Booij LHDJ, van Egmond $\mathrm{J}$ et al - Effect of isoflurane and sevoflurane on the magnitude and time course of neuromuscular block produced by vecuronium, pancuronium and atracurium. Br J Anaesth, 1996;76:389-395.

03. Suzuki T, Iwasaki K, Fukano N et al - Duration of exposure to sevoflurane effects dose-response relationship of vecuronium. Br J Anaesth, 2000;85:732-734.

04. Xue FS, Liao X, Tong SY et al - Dose-response and time-course of the effect of rocuronium bromide during sevoflurane anaesthesia. Anaesthesia, 1998;53:25-30.

05. Wulf H, Ledowski T, Linstedt U et al - Neuromuscular blocking effects of rocuronium during desflurane, isoflurane, and sevoflurane anaesthesia. Can J Anaesth, 1998;45:526-532.

06. Withington DE, Donati F, Bevan DR et al - Potentiation of atracurium neuromuscular blockade by enflurane: time-course of effect. Anesth Analg, 1991;72:469-473.

07. Ortiz JR, Percaz JA - Efecto de la técnica anestésica sobre la recuperación del bloqueo neuromuscular por cisatracurio. Rev Esp Anestesiol Reanim, 2001;48:117-121.

08. Braga AFA, Potério GMP, Braga FSS et al - Influência do sevoflurano e do isoflurano na duração do bloqueio neuromuscular produzido pelo rocurônio. Rev Bras Anestesiol, 2001;51:2-9.

09. Miller RD, Way WL, Dolan WM et al - Comparative neuromuscular effects of pancuronium, gallamine and succinylcholine during forane and halothane anesthesia in man. Anesthesiology, 1971;35:509-514.

10. Agoston S - Interactions of volatile anaesthetics with rocuronium bromide in perspective. Eur J Anaesth, 1994;11:(Supp19): 107-111.

11. Yasuda N, Lockhart SH, Eger EI Il et al - Comparison of kinetics of sevoflurane and isoflurane in humans. Anesth Analg, 1991; 72:316-324.

12. Yasuda N, Lockhart SH, Eger EI II et al - Kinetics of desflurane, isoflurane, and halothane in humans. Anesthesiology, 1991;74: 489-498.

13. Wulf $H$, Kahl $M$, Ledowski $T$ - Argumentation of the neuromuscular blocking effects of cisatracurium during desflurane, sevoflurane, isoflurane or total i.v. anaesthesia. $\mathrm{Br} \mathrm{J}$ Anaesth, 1998;80:308-312. 
14. Mallampati SR, Gatt SP, Gugino LD et al - A clinical sign to predict difficult tracheal intubation: a prospective study. Can J Anaesth, 1985;32:429-434.

15. Oris B, Crul JF, Vandermeersch E et al - Muscle paralysis by rocuronium during halothane, enflurane, isoflurane, and total intravenous anesthesia. Anesth Analg, 1993;77:570-573.

16. Shanks CA, Fragen RJ, Ling D - Continuous intravenous infusion balanced, enflurane, or isoflurane anesthesia. Anesthesiology, 1993;78:649-651.

17. Hemmerling TM, Shuettler J, Schwilden H - Desflurane reduces the effective therapeutic infusion rate (ETI) of cisatracurium more than isoflurane, sevoflurane, or propofol. Can J Anaesth, 2001:48:532-537.

18. Pollard BJ - Interactions Involving Relaxants, em: Pollard BJ Applied Neuromuscular Pharmacology, Oxford, Oxford University Press, 1994;202-228.

19. Vitez TS, Miller RD, Eger El II et al - Comparison in vitro of isoflurane and halothane potentiation of d-tubocurarine and succinylcholine neuromuscular blockades. Anesthesiology, 1974;41:53-56

20. Waud BE, Waud DR - The effects of diethyl ether, enflurane and isoflurane at the neuromuscular junction. Anesthesiology, 1975;42:275-280.

21. Waud BE - Decrease in dose requirements of d-tubocurarine by volatile anesthetics. Anesthesiology, 1979;51:298-302.

22. Brett RS, Dilger JP, Yland KF - Isoflurane causes "flickering" of the acetylcholine receptor channel: observations using the patch clamp. Anesthesiology, 1988;69:161-170.

23. Lowry DW, Mirakhur RK, Carroll MT et al - Potency and time course of mivacurium block during sevoflurane, isoflurane and intravenous anesthesia. Can J Anaesth, 1999;46:29-33.

24. Driessen JJ, Crul JF, Jansen $\mathrm{R}$ et al - Isoflurane and neuromuscular blocking drugs. Anaesth Int Care, 1986;182: 76-82.

25. Cannon JF, Fahey MR, Castagnoli KP et al - Continuous infusion of vecuronium: the effect of anesthetic agents. Anesthesiology, 1987;67:503-506

26. Shanks CA - Pharmacokinetics of the nondepolarizing neuromuscular relaxants applied to the calculation of bolus and infusion dosage regimens. Anesthesiology, 1986;64:72-86.

27. Hunter JM - New neuromuscular blocking drugs. N England $\mathrm{J}$ Med, 1995;332:1691-1699.

28. Fisher DM, Rosen $\mathrm{JI}$ - A pharmacokinetic explanation for increasing recovery time following larger or repeated doses of nondepolarizing muscle relaxants. Anesthesiology, 1986;65: 286-291.

29. Ginsberg B, Glass PS, Quill T et al - Onset and duration of neuromuscular blockade following high-dose vecuronium administration. Anesthesiology, 1989;71:201-205.

30. Bock M, Klippel K, Nitsche B et al - Rocuronium potency and recovery characteristics during steady-state desflurane, sevoflurane, isoflurane or propofol anaesthesia. $\mathrm{Br} \mathrm{J}$ Anaesth, 2000;84: 43-47.

31. Belmont MR, Lien CA, Quessy $S$ et al - The clinical neuromuscular pharmacology of 51W89 in patients receiving nitrous oxide/opioid/barbiturate anesthesia. Anesthesiology, 1995;82:1139-1145.

32. Lepage JY, Malinovsky JM, Malinge M et al - Pharmacodynamic dose response and safety study of cisatracurium (51W89) in adult surgical patients during $\mathrm{N}_{2} \mathrm{O}-\mathrm{O}_{2}$-opioid anesthesia. Anesth Analg, 1996;83:823-829.

33. Carpenter RL, Eger EI II, Johnson BH et al - Pharmacokinetics of inhaled anesthetics in humans: Measurements during and after the simultaneous administration of enflurane, isoflurane, methoxyflurane and nitrous oxide. Anesth Analg, 1986;65: 575-582.
34. Wright PMC, Hart P, Lau M et al - The magnitude and time course of vecuronium potentiation by desflurane versus isoflurane. Anesthesiology, 1995;82:404-411.

35.Gill SS, Bevan DR, Donati F - Edrophonium antagonism of atracurium during enflurane anaesthesia. $\mathrm{Br} \mathrm{J}$ Anaesth, 1990;64:300-305

36. Baurain MJ, d'Hollander AA, Melot $\mathrm{C}$ et al - Effects of residual concentrations of isoflurane on the reversal of vecuronium-induced neuromuscular blockade. Anesthesiology, 1991;74: 474-478.

\section{RESUMEN}

Braga AFA, Braga FSS, Potério GMB, Cremenosi E, Mauro G Influencia del Sevoflurano y del Isoflurano en la Recuperación del Bloqueo Neuromuscular Producido por el Cisatracúrio

Justificativa y Objetivos - Los efectos de los agentes bloqueadores neuromusculares sobre la juntura neuromuscular son aumentados por los anestésicos volátiles. El objetivo de este estudio fue evaluar la influencia del sevoflurano y del isoflurano en la recuperación del bloqueo neuromuscular producido por el cisatracúrio.

Método - Fueron estudiados 90 pacientes, estado físico ASA I y II, sometidos a cirugías electivas bajo anestesia general, distribuidos en tres grupos: Grupo I (sevoflurano), Grupo II (isoflurano) y Grupo III (propofol). Todos los pacientes recibieron como medicación pré-anestésica, midazolan $(0,1$ $\mathrm{mg} . \mathrm{kg}^{-1}$ ) por vía muscular, 30 minutos antes de la cirugía. La inducción anestésica fue obtenida con alfentanil $\left(50 \mu \mathrm{g} . \mathrm{kg}^{-1}\right)$, propofol $\left(2,5 \mathrm{mg}^{\mathrm{kg}} \mathrm{k}^{-1}\right)$ y cisatracúrio $\left(0,15 \mathrm{mg} \cdot \mathrm{kg}^{-1}\right)$. Los pacientes fueron ventilados con oxígeno a $100 \%$ bajo máscara hasta la desaparición de las cuatro respuestas a SQE, cuando fueron realizadas las maniobras de laringoscopia e intubación traqueal. Los agente volátiles para la manutención de la anestesia fueron introducidos luego después de la intubación traqueal y empleados en las concentraciones de $2 \%$ y $1 \%$, respectivamente para el sevoflurano e isoflurano, y el propofol en infusión continuada ( 7 a $\left.10 \mathrm{mg} \cdot \mathrm{kg}^{-1} \cdot \mathrm{h}^{-1}\right)$. En todos los pacientes se empleó la mezcla de oxígeno y óxido nitroso a $50 \%$. La función neuromuscular fue monitorizada por aceleromiografia del músculo aductor del pulgar, empleándose la SQE a cada 15 segundos. Fueron evaluados: la duración clínica del bloqueo neuromuscular $\left(T 1_{25 \%}\right)$ y el índice de recuperación (IR $\left.=T 1_{25-75 \%}\right)$.

Resultados - Los tiempos medios y desvíos patrón para la duración clínica $\left(T 1_{25 \%}\right)$ e índice de recuperación $\left(T 1_{25-75 \%}\right)$ fueron respectivamente: Grupo I (66,2 $\pm 13,42$ min y 23,6 $\pm 5,02$ min), Grupo II (54,4 $\pm 6,58 \mathrm{~min}$ y 14,9 $\pm 3,82 \mathrm{~min})$ y Grupo III $(47,2 \pm 7,43$ y 16,2 $\pm 2,93)$. En relación a la duración clínica hubo diferencia significante entre los grupos I y II, I y III, y II y III. Para el índice de recuperación hubo diferencia significante entre el grupo I y los demás grupos.

Conclusiones - La recuperación del bloqueo neuromuscular producido por el cisatracúrio fue más lenta durante la anestesia con los agentes volátiles de que con el propofol, siendo el efecto más pronunciado con el sevoflurano. 